\section{Is There Unwarranted Risk in Cohorting AIDS Patients?}

\section{To the Editor:}

Doctors Hospital is a voluntary community institution of 263 beds located in New York City. We are currently undergoing growing pains in the form of a multimillion dollar renovation and expansion project. Upon completion there will be completely modernized patient floors, new clinical/pathology laboratories, operating rooms, radiology department and an expanded emergency room. In the midst of this activity the hospital has remained open and continues to serve the surrounding Yorkville Community. Through repeated emphasis of basic sanitarian principles and infection control practices our nosocomial infection rate has remained relatively stable with no incidents traceable to construction activity (eg, Aspergillosis in immunocompromised patients).

A situation arose which required some reflection on my part. To provide a properly functioning independent ventilation system for our isolation rooms required their being out of service for 3 to 4 days. Our isolation rooms are vertically aligned with one on each of seven patient floors. While ideally they are reserved for diseases which necessitate separation such as those under respiratory, $\mathrm{AFB}$ and contact isolation, they are more often used for individual AIDS patients. We acknowledge that these patients usually need be on Blood/Body Fluid Precautions only as per CDC Guidelines. ${ }^{1,2}$ However, the anxiety elicited by posting the obligatory sign is significant. The alarm felt by the roommate and his family/friends is such that it is often simpler to use the isolation rooms when available. It is understood however that should a patient be admitted with a more communicable disease (eg, tuberculosis, meningitis), the AIDS patient will be bumped to a private room at the hospital's expense.

Having to keep our isolation rooms empty for 3 to 4 days raised the question of whether or not AIDS patients could be cohorted in two bedded, semi-private rooms. This would leave the private rooms available for those patients wishing to pay for such accommodations. As with many other New York Hospitals we have recently experienced a dramatic increase in the number of AIDS cases. In 1982 we saw perhaps one case every 3 months. Currently we average five cases in any given week which therefore ties up five of our seven isolation rooms.

When I was asked whether it was feasible to cohort our AIDS patients, my initial thought was why not, assuming of course, the patients were carefully selected. For example under no circumstances should an AIDS victim with Pneumocystis carinii pneumonia (PCP) be roomed with another who is free of this opportunistic infection. In essence I found myself attempting to pair AIDS patients based on specific opportunistic infections present and the degree of debilitation. For example, two patients with PCP and oral thrush might be considered excellent choices for roommates. On the other hand, with patients as vulnerable as AIDS victims, I was not comfortable with this idea. With the severely immunosuppressed we cannot anticipate all changes. Although when initially roomed together patients may have matching opportunistic infections we cannot predict when one of them may develop additional infections such as reactivated CMV or profuse diarrhea due to cryptosporidiosis. By the time such pathogens are detected it may be too late to protect the susceptible roommate. In spite of the fact that many opportunistic infections are transmissible only through direct contact there is still a high risk of cross-infection. How many health care workers wash their hands between patients within the same room?

The situation is not similar to cohorting two or more patients with the same strain of bacterial or viral pneumonia. With AIDS we are dealing with a syndrome that makes the victim vulnerable to a wide variety of opportunistic infections often endogenous in origin. Therefore we cannot predict which opportunistic infection will surface in a patient nor when.

As one of the primary roles of an Infection Control Practitioner is prevention, it would appear to be prudent to avoid cohorting AIDS victims when possible.

Lastly, these are times of profuse litigation. It would be exceedingly difficult to explain to a lay jury our rationale of a policy which may easily lead to a life-threatening infection in an already debilitated patient.

\section{REFERENCES}

1. Centers for Disease Control, Bureau of Epidemiology. Acquired immunodeficiency syndrome (AIDS): Precautions for clinical and laboratory staffs. MMWR 1982; $31(43): 577-580$.

2. Centers for Disease Control, Bureau of Epidemiology: Acquired immunodeficiency syndrome (AIDS): Precautions for health care workers and allied professionals. MMWR 1983; 32(34):450-451.

Charles B. Burrus, MS, CIC Infection/Environmental Control Coordinator Doctors Hospital New York, New York

\section{Group A Streptococcal Pharyngitis in Hospital Personnel}

\section{To the Editor:}

It is with great interest that we read "Should routine throat cultures be done in hospital personnel complaining of a sore throat?" 1 since we have also been concerned about this problem in our hospital for the past several years.

We are a metropolitan teaching hospital with over 800 beds and approximately 4,300 employees from 1982 to 1985. During this period we have performed throat cultures on almost all 